\title{
Effect of cohesion on shear banding in quasistatic granular materials
}

\author{
Abhinendra Singh, ${ }^{*}$ Vanessa Magnanimo, Kuniyasu Saitoh, and Stefan Luding \\ Multi Scale Mechanics (MSM), Faculty of Engineering Technology, MESA+, University of Twente, P. O. Box 217, \\ 7500 AE Enschede, The Netherlands
}

(Received 26 December 2013; published 6 August 2014)

\begin{abstract}
Cohesive powders have widely different bulk behavior due to their peculiar interactions. We use discrete element simulations to investigate the effect of contact cohesion on the steady state flow of dense powders in a slowly sheared split-bottom Couette cell, which imposes a wide stable shear band. The intensity of cohesive forces can be quantified by the granular Bond number (Bo), namely the ratio between maximum attractive force and average force due to external compression. We find that the shear banding phenomenon is almost independent of cohesion for Bond numbers Bo $<1$, however for Bo $\geqslant 1$ cohesive forces start to play an important role, as both width and center position of the band increase. Inside the shear band, the mean normal contact force is independent of cohesion and depends only on the confining stress. In contrast, when the behavior is analyzed focusing on the eigendirections of the local strain rate tensor, a dependence on cohesion shows up. Forces carried by contacts along the compressive and tensile directions are symmetric about the mean force (larger and smaller respectively), while the force along the third, neutral direction follows the mean force. This anisotropy of the force network increases with cohesion, just like the heterogeneity in all (compressive, tensile and neutral) directions.
\end{abstract}

DOI: 10.1103/PhysRevE.90.022202

PACS number(s): 45.70.Mg, 83.50.Ax, 83.85.Cg

\section{INTRODUCTION}

Granular materials such as sand and limestone behave neither like elastic solids nor like normal fluids, which makes their motion difficult to predict. When they yield under slow shear, the relative motion is confined to narrow regions (between large solidlike parts) called shear bands [1-3]. Shear bands are observed in many complex materials, which range from foams [4] and emulsions [5,6] to colloids [7] and granular matter [1,2,8-17]. There has been tremendous effort aimed towards understanding shear bands in flow of noncohesive grains [1,2,8-19]. However, real granular materials often experience interparticle attractive forces due to many physical phenomena: van der Waals due to atomic forces for small grains [20-22], capillary forces due to presence of humidity [23], solid bridges [24,25], coagulation of particles [26], and many more.

The question arises regarding how the presence of attractive forces affects shear banding. So far, only a few attempts have been made to answer this question concerning dense metallic glasses [27,28], adhesive emulsions [29,30], attractive colloids [31-33], cemented granular media [34], wet granular media $[35,36]$, and clayey soils [37]. Recently, rheological studies on adhesive emulsions and colloids [29-31,33] showed that the presence of attractive forces at contact affects shear banding by affecting flow heterogeneity and wall slip.

Another unique yet not completely understood feature of granular materials is their highly heterogeneous contact force distribution, as observed in both experimental and numerical studies [2,38-46]. While huge effort has been made to understand the force distribution of noncohesive particles [2,38$44,47]$, only limited studies have aimed to understand the same for assemblies with attractive interactions [21,48-52]. Richefeu et al. [49] studied the stress transmission in a wet

\footnotetext{
*a.singh-1@utwente.nl
}

granular system subjected to isotropic compression. Gilabert et al. [50] focused on a two-dimensional packing made of particles with short-range interactions (cohesive powders) under weak compaction. Yang et al. [51] studied the effect of cohesion on force structures in a static granular packing by changing the particle size. In a previous study [53], the effect of dry cohesion at contact on the critical state yield stress was studied. The critical-state yield stress shows a peculiar nonlinear dependence on the confining pressure related to cohesion. But the microscopic origin has not been studied.

In this paper, we report the effect of varying the strength of attractive forces at contact on the steady-state flow behavior and the force structure in slowly sheared dry cohesive powders. Discrete element method (DEM) simulations are used to investigate the system at the micro (partial) and macro levels. In order to quantify the intensity of cohesion, a variation of the granular Bond number (Bo) $[50,54,55]$ is introduced. We find that this dimensionless number very well captures the transition from a gravity- and shear-dominated regime to a cohesion-dominated regime. To understand this further we look at the effect of cohesion on the mean force and anisotropy by investigating the forces along the eigendirections of the local strain rate tensor. Intuitively, one would expect only the tensile direction to be affected by cohesion, but the real behavior is more complex. We also discuss the probability distributions and heterogeneities of the forces in different directions for a complete picture.

The paper is organized in four main parts. Section II describes the model system in detail specifying the geometry, details of particle properties, and the interaction laws. In Sec. III, the velocity profiles and shear band from samples with different contact cohesion are presented. In the same section, the force anisotropy and probabilities are studied, too. Finally, Sec. IV is dedicated to the discussion of the results, conclusions and an outlook. 


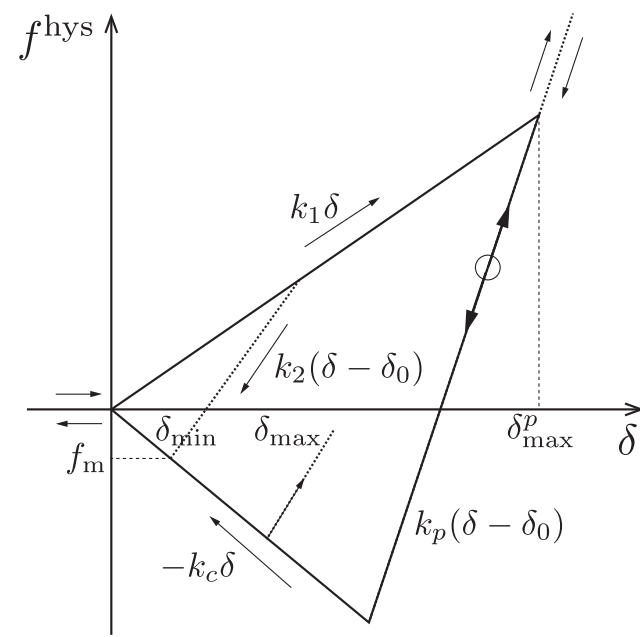

FIG. 1. Schematic graph of the piecewise linear, hysteretic, and adhesive force-displacement model in normal direction.

\section{DISCRETE ELEMENT METHOD SIMULATION}

In this section, we explain our DEM simulations. We introduce a model of cohesive grains in Sec. II A and show our numerical setup in Sec. II B. In Sec. II C, we propose a control parameter, i.e., the global Bond number, which governs the flow profiles and structure of the system.

\section{A. Model}

DEM provides numerical solutions of Newton's equations of motion based on the specification of particle properties viz. stiffness, density, radius, and certain interaction laws like Hertzian and Hookean [56,57]. Simulation methodology and material parameters used in this study are the same as in our previous work $[53,58]$. The adhesive elastoplastic contact model [59] is used to simulate cohesive bulk flow, as briefly explained below.

For fine, dry powders, adhesive properties due to van der Waals forces and plasticity and irreversible deformation in the vicinity of the contact have to be considered at the same time $[60,61]$. This complex behavior is modeled using a piecewise linear hysteretic spring model [59] (Fig. 1). Few other contact models in similar spirit were recently proposed [62,63].

The adhesive, plastic (hysteretic) force is introduced by allowing the normal unloading stiffness to depend on the history of deformation. During initial loading the force increases linearly with overlap $\delta$ along $k_{1}$, until the maximum overlap $\delta_{\text {max }}$ is reached, which acts as a history parameter. During unloading the force decreases along $k_{2}$, the value of which depends on the maximum overlap $\delta_{\max }$ as given by Eq. (2). The overlap when the unloading force reaches zero, $\delta_{0}=\left(1-k_{1} / k_{2}\right) \delta_{\max }$, resembles the permanent plastic deformation and depends nonlinearly on the previous maximal force $f_{\max }=k_{1} \delta_{\max }$. The negative forces reached by further unloading are attractive, cohesion forces, which also increase nonlinearly with the previous maximum force experienced. The maximal cohesion force that corresponds to the pull-off force, is given by

$$
f_{\mathrm{m}}=-k_{c} \delta_{\min },
$$

with $\delta_{\min }=\frac{k_{2}-k_{1}}{k_{2}+k_{c}} \delta_{\max }$.
Three physical phenomena, elasticity, plasticity, and cohesion, are quantified by three material parameters, $k_{p}, k_{1}$, and $k_{c}$, respectively. Plasticity disappears for $k_{1}=k_{p}$ and cohesion vanishes for $k_{c}=0$. In the following we focus on the relative importance of cohesion and thus do not provide measurable force magnitudes. Furthermore, the contact model has to be seen as a mesoscale model, where each particle represents an ensemble of primary particles and the contact model represents the respective bulk behavior, see Ref. [64], without a direct match of the magnitude of forces in the model with the forces between the primary particles. Qualitatively, the interpretation of $k_{c}$ is that it describes the increased van der Waals type adhesion due to plastic deformations (both of the particles and the microstructure) under compression, which increases the contact surface and thus the cohesion. Some considerations on the magnitude and relative importance of the cohesion force can be found in Appendix B.

In order to account for realistic load-dependent contact behavior, the $k_{2}$ value is chosen to depend on the maximum overlap $\delta_{\max }$, i.e., particles are more stifffor larger previous deformation and so the dissipation is dependent on deformation. The dependence of $k_{2}$ on overlap $\delta_{\max }$ is chosen empirically as linear interpolation,

$$
k_{2}\left(\delta_{\max }\right)=\left\{\begin{array}{lll}
k_{p} & \text { if } & \delta_{\max } / \delta_{\max }^{p} \geqslant 1 \\
k_{1}+\left(k_{p}-k_{1}\right) \frac{\delta_{\max }}{\delta_{\max }^{p}} & \text { if } & \delta_{\max } / \delta_{\max }^{p}<1
\end{array} .\right.
$$

As discussed in Ref. [59], very large deformations will lead to a quantitatively different contact behavior, a maximal force overlap $\delta_{\max }^{p}=\frac{k_{p}}{k_{p}-k_{1}} \frac{2 a_{1} a_{2}}{a_{1}+a_{2}} \phi_{f}$ is defined (with $\phi_{f}=0.05$ ). Above this overlap $k_{2}$ no longer increases and is set to the maximal value $k_{2}=k_{p}$. This viscoelastic, reversible branch is referred to as the limit branch.

The contact friction is set to $\mu=0.01$, i.e., artificially small, in order to be able to focus on the effect of contact cohesion only. We analyzed the system for the following set of adhesivity parameters $k_{c}$ :

$$
k_{c} \in[0,5,10,25,33,50,75,100,200] \mathrm{Nm}^{-1},
$$

which has to be seen in relation to $k_{1}=100 \mathrm{Nm}^{-1}$. Other parameters, such as the jump-in force $f_{a}=0$ [64] and $\phi_{f}=$ 0.05 [64], are not varied here. We also introduce damping forces proportional to the normal and tangential relative velocities, where the viscous coefficients are given by $\gamma_{n}=$ $0.002 \mathrm{~s}^{-1}$ and $\gamma_{t}=0.0005 \mathrm{~s}^{-1}$, respectively.

\section{B. Split-bottom ring shear cell}

Figure 2 is a sketch of our numerical setup (as introduced in Refs. [15,65-68]). In this figure, the inner, split, and outer radii are given by $R_{i}, R_{s}$, and $R_{o}$, respectively, where the concentric cylinders rotate relative to each other around the symmetry axis (the dot-dashed line). The ring shaped split at the bottom separates the moving and static parts of the system, where a part of the bottom and the outer cylinder rotate at the same rate. The system is filled with $N \approx 3.7 \times 10^{4}$ spherical particles with density $\rho=2000 \mathrm{~kg} / \mathrm{m}^{3}=2 \mathrm{~g} / \mathrm{cm}^{3}$ up to height $H$. The average size of particles is $a_{0}=1.1 \mathrm{~mm}$, and the width of the homogeneous size distribution (with $a_{\min } / a_{\max }=1 / 2$ ) is $1-\mathscr{A}=1-\langle a\rangle^{2} /\left\langle a^{2}\right\rangle=0.18922$. The cylindrical walls 


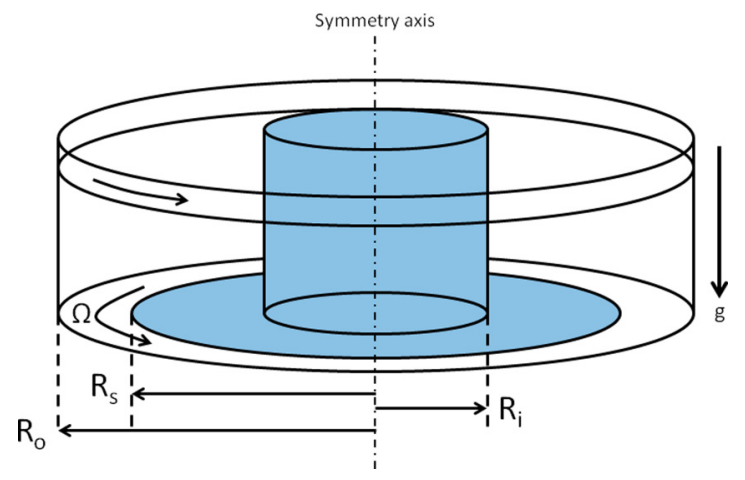

FIG. 2. (Color online) A sketch of our numerical setup consisting of a fixed inner part (light blue shade) and a rotating outer part (white). The white part of the base and the outer cylinder rotate with the same angular velocity $\Omega$ around the symmetry axis. The inner, split, and outer radii are given by $R_{i}=0.0147 \mathrm{~m}, R_{s}=0.085 \mathrm{~m}$, and $R_{o}=0.11 \mathrm{~m}$, respectively, where each radius is measured from the symmetry axis. The gravity $g$ points downwards as shown by arrow.

and the bottom are roughened due to some (about $3 \%$ of the total number) attached and glued particles [66,67].

When there is a relative motion at the split, a shear band propagates from the split position $R_{s}$ upwards and inwards, remaining far from the cylinder walls and bottom in most cases. The qualitative behavior is governed by the ratio $H / R_{s}$ and three different regimes can be identified, as reported in Refs. [68-71]. We keep $H / R_{s}<0.5$, such that the shear band reaches the free surface and stays away from the inner wall $[69,70]$.

Translational invariance is assumed in the azimuthal $\theta$ direction, and the averaging is performed over toroidal volumes, over many snapshots in time. This leads to fields $Q(r, z)$ as function of the radial and vertical positions.

Since we are interested in the quasistatic regime, the rotation rate of the outer cylinder is chosen to be $0.01 \mathrm{~s}^{-1}$, such that the inertial number $I=\frac{\dot{\gamma} d}{\sqrt{p / \rho}}[72]$ is $I \ll 1$, and the simulation runs for more than $50 \mathrm{~s}$.

\section{Bond number}

Intensity of cohesion can be quantified by the ratio of the maximum attractive force to a typical force scale in the system. For example, Nase et al. [54] introduced the granular Bond number under gravity, which compares the maximum attractive force at contact with the weight of a single grain. For plane shear without gravity, other authors $[50,55]$ used a ratio between the maximum attractive force and the average force due to the confining pressure. In our analysis, we introduce a global Bond number as

$$
\mathrm{Bo}=\frac{f_{\mathrm{m}}}{\langle f\rangle},
$$

where $f_{\mathrm{m}}$ and $\langle f\rangle$ are the maximum allowed attractive force reached at a contact (given by the contact model, see Appendix A, using $\delta_{\max }=\delta_{\max }^{p}$ ) and the mean force per contact reached close to the bottom, respectively. For the calculation of the mean force $\langle f\rangle$, a layer two particle diameters from the bottom is chosen, as the shear band initiates

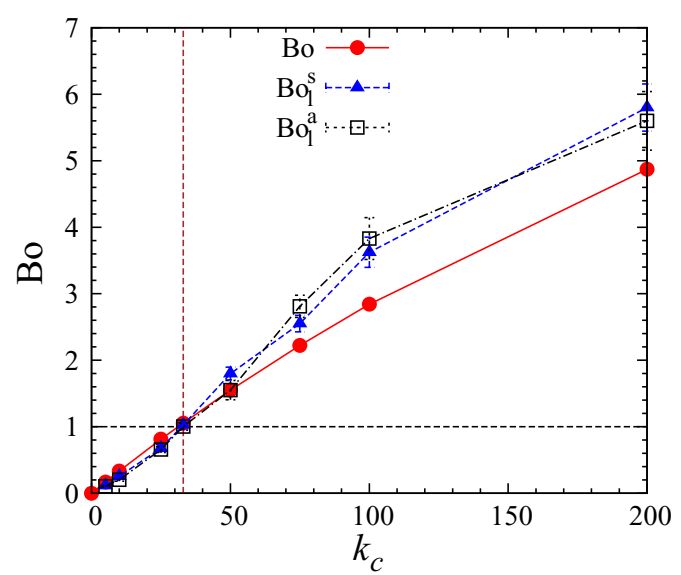

FIG. 3. (Color online) Variants of granular Bond number plotted against cohesive strength $k_{c}$, where the red circles represent the global Bond number Bo, while the blue triangles and black squares represent the average values of $\mathrm{Bo}_{l}^{s}(p)$ and $\mathrm{Bo}_{l}^{a}(p)$, respectively.

from the bottom, we choose the mean force $\langle f\rangle$ close to the bottom to understand the effect of cohesion on these shear bands.

It is important to mention that the mean force (at the bottom) corresponds to the weight of the material above, whereas the maximum attractive force corresponds to the pull-off force, which is directly related to the surface energy of the particles. These two material and particle properties are easily accessible experimentally, see Appendix B.

The Bond number is a measure of the relative importance of adhesive forces compared to external (compressive) force. A low Bond number indicates that the system is relatively unaffected by attractive forces; a high number (typically larger than 1) indicates that attractive forces dominate. Intermediate numbers indicate a nontrivial competition between the two effects.

In parallel with the global Bond number, we also define two local variants of this quantity. A local simulation-based Bond number $\mathrm{Bo}_{l}^{s}(p)=f_{\mathrm{m}}^{s}(p) /\langle f(p)\rangle$ can be defined by comparing the maximum attractive force reached at a given pressure (which can be less than or equal to the maximum allowed attractive force given by the contact model) with the mean force at that pressure (subscript $l$ represents the local quantity, while superscript $s$ denotes that this definition takes input from simulation data). Another variant of this $\mathrm{Bo}_{l}^{a}(p)$ is defined in Appendix A, which compares the analytical prediction for the maximum attractive force with the mean force at that pressure and does not use the gravitational Bond number, see Appendix B, since it is only relevant close to the free surface and for single particles in contact with a wall.

Figure 3 displays the global Bond number $\mathrm{Bo}$ and the mean values of $\mathrm{Bo}_{l}^{s}(p)$ and $\mathrm{Bo}_{l}^{a}(p)$ (averaged over different pressure) as functions of the adhesivity parameter $k_{c}$, where the figure shows that local and global quantities are comparable with slight differences for high cohesion $k_{c}$. For the sake of simplicity, in the rest of this paper, we use the global Bond number Bo to quantify the intensity of cohesion. 

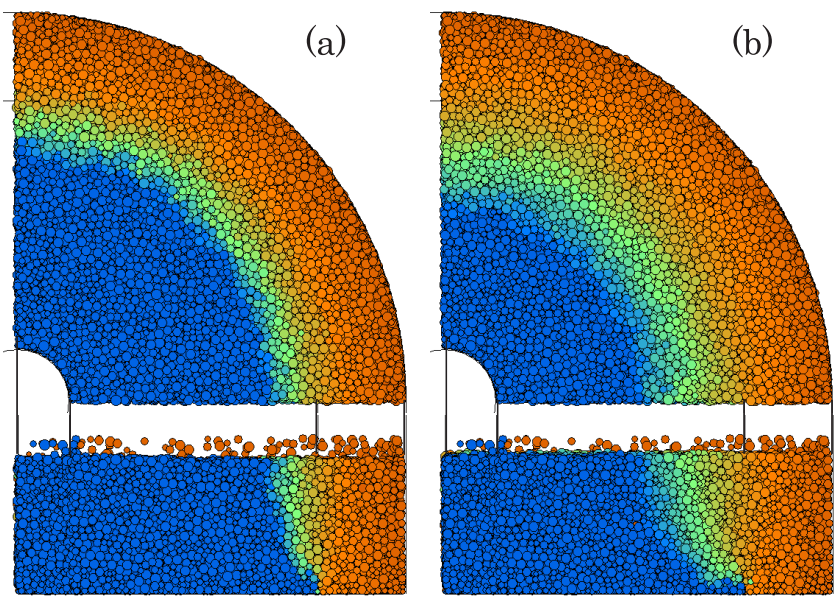

FIG. 4. (Color online) Snapshots from simulations with different cohesion strengths, but the same number of mobile particles $N=$ 34518 , seen from the top (top) and the front (bottom). The material is (a) without cohesion $\mathrm{Bo}=0$ and (b) with strong cohesion $\mathrm{Bo}=$ 4.86. Blue, green, and orange denote the particles with displacements in tangential direction per second $r d \phi \leqslant 0.5 \mathrm{~mm}, r d \phi \leqslant 2 \mathrm{~mm}$, $r d \phi \leqslant 4 \mathrm{~mm}$, and $r d \phi>4 \mathrm{~mm}$, respectively.

\section{RESULTS}

In this section, we present our results of DEM simulations. In Sec. III A, we analyze the flow profiles and shear banding in the system. In Sec. III B, we study distributions and structures of force chain networks in shear bands. In Sec. III C, we explain anisotropic features of the force chain networks.

\section{A. Effect of cohesion on flow profiles}

Figure 4 displays both top and front views of samples with the same filling height, i.e., same number of particles, and different global Bond numbers, Bo $=($ left) 0 and (right) 4.86, respectively, where the color code represents the azimuthal displacement rate of the particles. From the front view, we observe that the shear band (green) moves inwards and gets wider with increasing height and Bond number.

With the goal to extract quantitative data for the shear band area, in Fig. 5 we plot the nondimensional angular velocity profiles at the top surface against radial coordinate normalized with the mean particle diameter $\langle d\rangle$, where we assume translational invariance in the azimuthal direction and take averages over the toroidal volumes as well as many

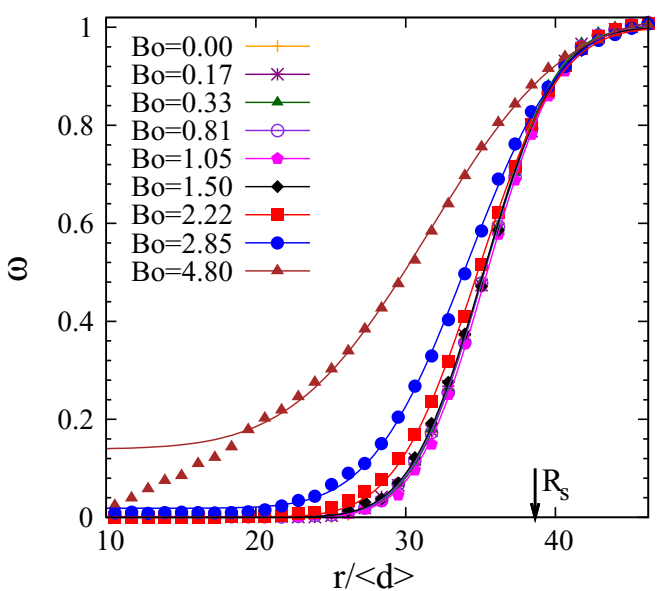

FIG. 5. (Color online) Nondimensional angular velocity profile $\omega$ at the top surface plotted against the radial coordinate $r$ scaled by the mean diameter $\langle d\rangle$. Different symbols represent different values of the global Bond number Bo given in the inset, where the solid lines represent the corresponding fits to Eq. (5).

snapshots in time [13]. The angular velocity profile can be well approximated by an error function,

$$
\omega=A_{1}+A_{2} \operatorname{erf}\left(\frac{r-R_{c}}{W}\right),
$$

as in the case of noncohesive materials [15,65-68], where $R_{c}$ and $W$ are the position and width of the shear band, respectively. Here we use the dimensionless amplitudes, $A_{1}=$ $A_{2} \approx 0.5$, for the whole range of the Bond numbers, while we use $A_{1}=0.6$ and $A_{2}=0.4$ for the strong cohesion with Bo $=4.86$. The dimensionless amplitudes, $A_{1}$ and $A_{2}$ (along with estimated errors), are summarized in Table I. Then we extract the position of the shear band relative to the split at the bottom $R_{s}-R_{c}$ and the width of the shear band $W$ (both scaled by mean particle diameter) at the top surface and we plot them in Fig. 6 against the Bond number. Within the error bars, both the position and width are independent of cohesion if $\mathrm{Bo}<1$. However, the shear band moves inside and becomes wider with increasing Bond number for Bo $>1$.

Both $R_{s}-R_{c}$ and $W$ also depend on the height $(z)$ in the system. Figure 7 displays the nondimensional position and width of the shear band for different values of Bo as functions of the height scaled by the filling height, i.e., $z / H$. The shear band moves closer to the inner cylinder

TABLE I. Table showing filling height of the system $H$ and fitting range $z / H$ for Eqs. (6) and (7), together with the fit parameters $A_{1}, A_{2}$ in Eq. (5), $\beta$ in Eq. (6), $W_{\text {top }}$, and $\gamma$ in Eq. (7) for different values of Bond number Bo.

\begin{tabular}{llccccc}
\hline \hline Bo & $A_{1}$ & $A_{2}$ & $H$ & $\beta$ & $\frac{z}{H}$ range & $W_{\text {top }}$ \\
\hline 0 & $0.50 \pm 0.0005$ & $0.500 \pm 0.0005$ & 0.0365 & 2.52 & $0.1-1$ & 0.0117 \\
0.17 & $0.50 \pm 0.0005$ & $0.499 \pm 0.0005$ & 0.0365 & 2.52 & $0.1-1$ & 0.0118 \\
0.33 & $0.49 \pm 0.0007$ & $0.500 \pm 0.0007$ & 0.0365 & 2.512 & $0.1-1$ & 0.507 \\
0.81 & $0.49 \pm 0.0008$ & $0.500 \pm 0.0008$ & 0.0361 & 2.494 & $0.1-1$ & 0.0118 \\
1.05 & $0.49 \pm 0.001$ & $0.501 \pm 0.001$ & 0.0359 & 2.510 & $0.1-1$ & 0.0119 \\
1.50 & $0.49 \pm 0.002$ & $0.501 \pm 0.002$ & 0.0364 & 2.453 & $0.1-0.8$ & 0.0120 \\
2.22 & $0.49 \pm 0.003$ & $0.501 \pm 0.003$ & 0.0368 & 2.367 & $0.1-0.6$ & 0.0126 \\
2.85 & $0.49 \pm 0.005$ & $0.502 \pm 0.005$ & 0.0369 & 2.259 & $0.1-0.6$ & 0.0138 \\
\hline \hline
\end{tabular}




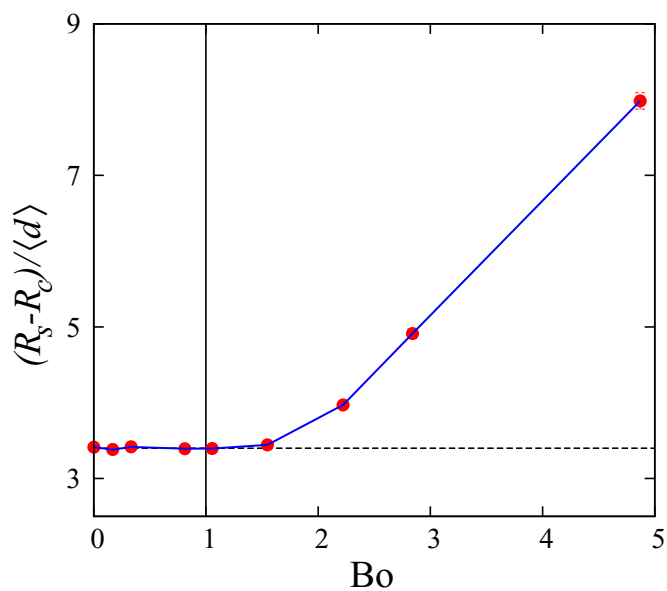

(a)

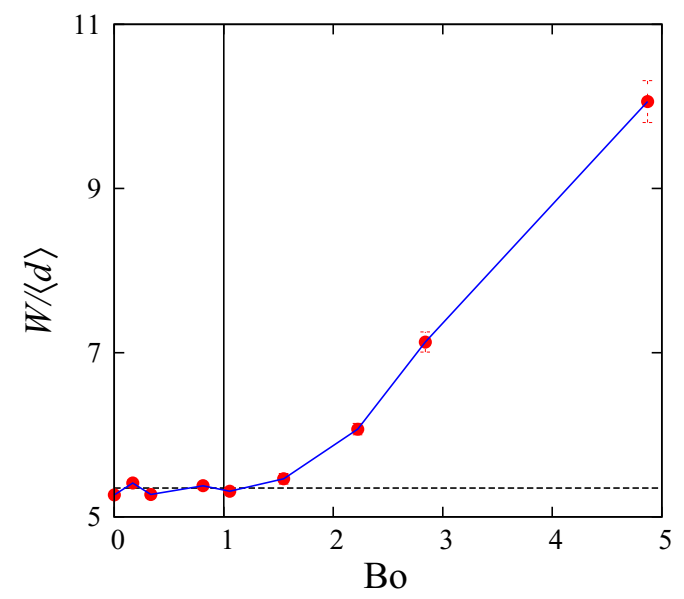

(b)

FIG. 6. (Color online) (a) Position and (b) width (both scaled by mean particle diameter) of the shear band at the top surface plotted against the global Bond number Bo. Symbols with error bars are the data, while the lines are only a guide to eye.

and gets wider while approaching the top layer, which is consistent with previous studies [15,53,65-68,71] on cohesive and noncohesive assemblies. In Fig. 7(a), the lines are the prediction by Unger et al. [69],

$$
z=H-R_{c}\left\{1-\frac{R_{s}}{R_{c}}\left[1-\left(\frac{H}{R_{s}}\right)^{\beta}\right]\right\}^{1 / \beta},
$$

where the exponent is given by $\beta=2.5$ for noncohesive particles. If the Bond number is less than 1, the data collapse on a unique curve, very well predicted by Eq. (6), with fixed exponent $\beta$. On the other hand, above $\mathrm{Bo}=1$, the exponent $\beta$ decreases with the global Bond number (values reported in Table I). Note that Eq. (6) slightly deviates from the results near the top surface if the cohesion is strong $(\mathrm{Bo}=2.22$ and 2.85). In Fig. 7(b), the lines are the prediction by Ries et al. [71] for a noncohesive system,

$$
W(z)=W_{\text {top }}\left[1-\left(1-\frac{z}{H}\right)^{2}\right]^{\gamma},
$$

where $W_{\text {top }}$ is the width at the top surface and the exponent is given by $\gamma=0.5$ for noncohesive particles. If $\mathrm{Bo}<1$,

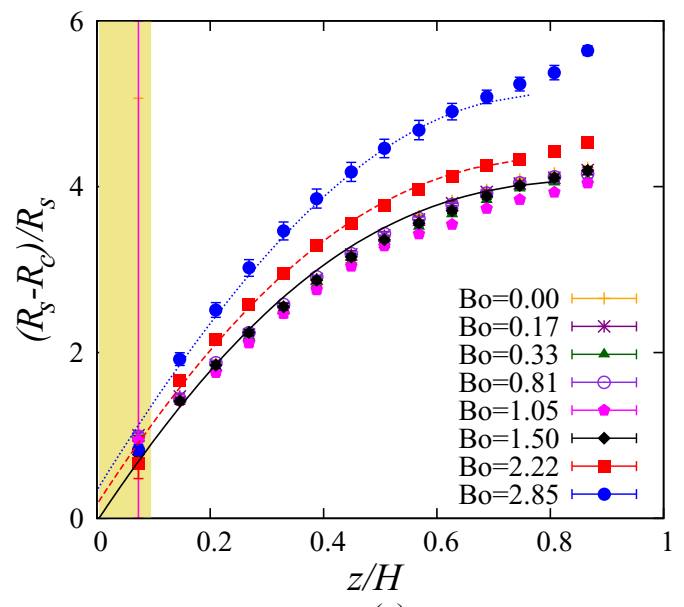

(a)

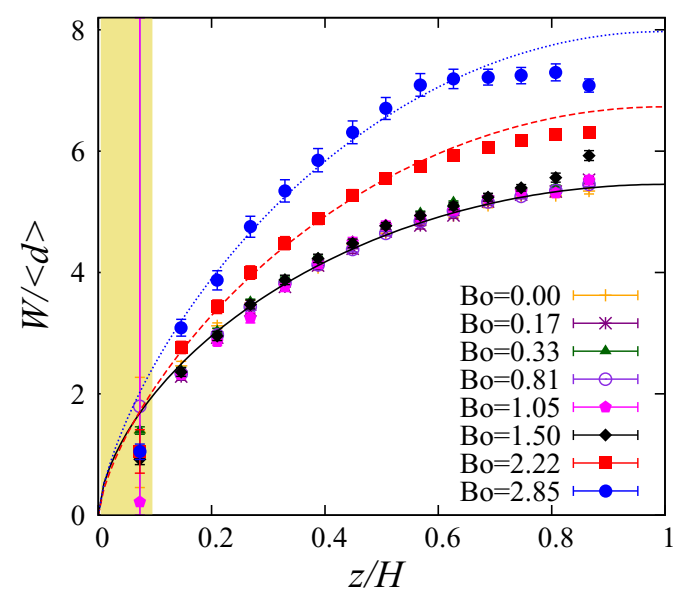

(b)

FIG. 7. (Color online) (a) Position and (b) width (both scaled by mean particle diameter) of shear band in the cell plotted against height $z$ scaled by the filling height $H$. Different symbols correspond to values of the global Bond number Bo given in the inset. The lines in (a) and (b) are the predictions, Eqs. (6) and (7), respectively.

Eq. (7) with $W_{\text {top }}=0.012$ and $\gamma=0.5 \pm 0.1$ well agrees with our results. However, for Bo $>1$, both the width $W_{\text {top }}$ and exponent $\gamma$ increase with the global Bond number as in Table I. In addition, Eq. (7) deviates from the results near the top layer if the cohesion is strong $(\mathrm{Bo}=2.22$ and 2.85), where $W$ seems to saturate above $z / H \simeq 0.6$. Hence for $\mathrm{Bo}>1$, we choose the width at that height to be $W_{\text {top }}$ and use $\gamma=0.66$ and 0.7 for $\mathrm{Bo}=2.22$ and 2.85 , respectively.

From the above results, we conclude that the cohesive forces between particles drastically affect the flow profiles. Equations (6) and (7) predict the position and width of the shear bands for Bo $<1$ very well. For large Bo these equations deviate from observed behavior at large heights since the shear band interferes with the inner cylinder. The shear band, which is the region with a large velocity gradient, is caused by sliding motions of particles. However, strong cohesive forces keep particles in contact (in other words, the cohesive forces promote collective motions of particles) and prevent them from sliding. As a result, the velocity gradient is smoothened and the width of the shear band is broadened. This observation is 
consistent with previous studies on adhesive dense emulsions [73]. Interestingly, such an effect of cohesion is suppressed if the global Bond number is less than 1, where our numerical data agrees well with previous theoretical/numerical studies on noncohesive particles [69,71]. Hence, the global Bond number, Bo, captures the transition between essentially noncohesive free-flowing granular assemblies $($ Bo $<1)$ to cohesive ones $(\mathrm{Bo}>1)$.

\section{B. Structure and distribution of forces in shear bands}

To understand the microscopic origin of the anomalous flow profiles of cohesive aggregates, we study the force network and the statistics of the Interparticle normal contact forces. Recently Wang et al. [74] reported the shape of the probability distribution function (PDF) as an indicator for the transition from quasistatic to inertial flows for noncohesive particles. In this section, we use a similar philosophy and study the change in the shape of PDFs as the cohesive strength is increased.

Figure 8 shows force chains of positive [Figs. 8(a) and 8(b)] and negative [Figs. 8(c) and 8(d)] normal forces in the systems with low cohesion [Figs. 8(a) and 8(c)] and strong cohesion [Figs. 8(b) and 8(d)]. Gray shows the weak forces, while red and blue show the strong positive and negative forces, respectively. The strong or weak positive forces are forces larger or smaller than the mean positive force $f_{\text {pos }}$. A similar approach is adopted to identify the strong and weak negative forces. In this figure, we observe that both positive and negative

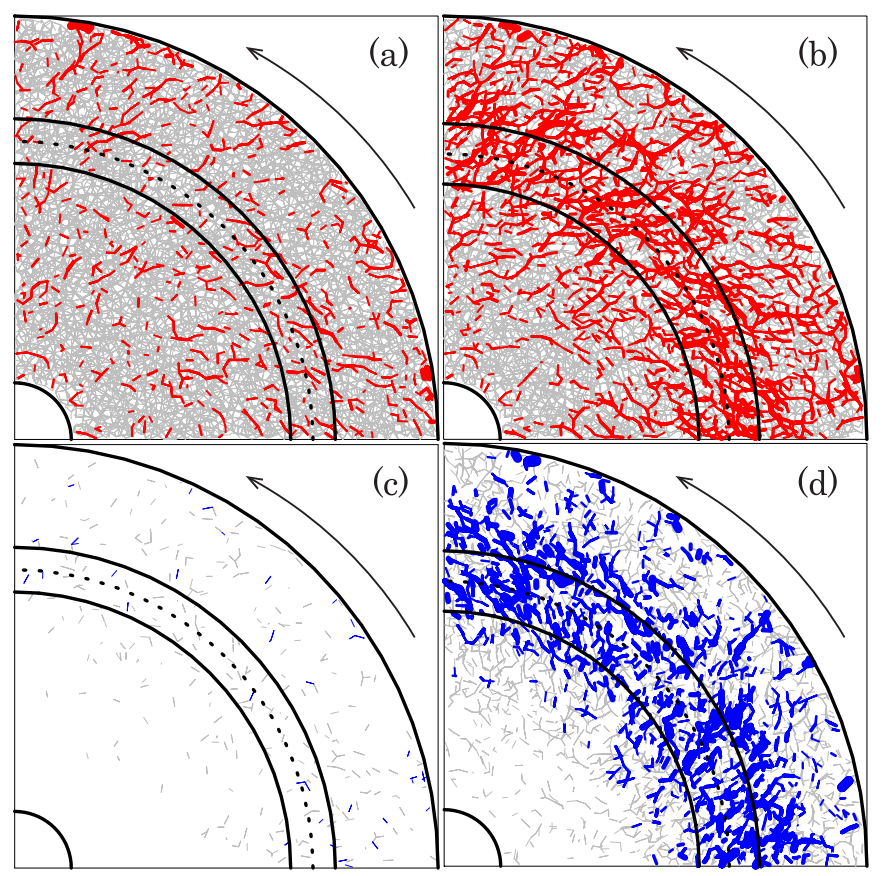

FIG. 8. (Color online) Force chain networks of positive normal forces for $\mathrm{Bo}=0.33$ (a) and 2.85 (b), and negative normal forces for $\mathrm{Bo}=0.33$ (c) and 2.85 (d) at height $0.02<z<0.025 \mathrm{~m}$, respectively. In (a) and (b) positive normal force smaller than $0.002 \mathrm{~N}$ is represented by grey, while larger than $0.002 \mathrm{~N}$ is represented by red color. In (c) and (d) negative normal force smaller than $-0.0005 \mathrm{~N}$ is represented by gray, while larger than $-0.0005 \mathrm{~N}$ is represented by blue color. forces are fully developed in the cohesive system [Figs. 8(b) and $8(\mathrm{~d})$ ], with the intensity of the force inside the shear band being stronger than outside. In addition, the strong (positive or negative) force chains are percolated through the shear band region.

Figure 9 displays scatter plots of the interparticle forces against overlaps between the particles in contacts, where each point corresponds to a contact and different colors represent different heights, i.e., pressure levels in the system. The higher the pressure $p$, the higher the average force (or overlap), as it must sustain the weight of the particles. For almost all values of Bo, the density of points towards the unloading branch $k_{p}$ is higher inside the shear band compared to outside. We also observe that with increasing Bo, most contacts (except for small pressure) drift towards and collapse around the limit branch. This implies that the cohesive forces are more pronounced in shear bands rather than outside.

\section{Mean force and overlap in shear bands}

Figure 10 displays the mean normal forces, $\langle f\rangle$, in the shear band plotted against pressure for different values of the global Bond number, where the solid line is the prediction by Shaebani et al. [75] for noncohesive granular systems,

$$
\langle f\rangle=\frac{4 \pi\left\langle a^{2}\right\rangle}{\phi C g_{2}}\langle p\rangle
$$

with the second moment of the size distribution $\left\langle a^{2}\right\rangle$, coordination number $C$, volume fraction $\phi$, and mean pressure $\langle p\rangle$. Notably, the mean normal force is almost independent of cohesion and linearly increases with pressure as in the cases of static noncohesive $[39,45]$ and cohesive systems [51]. We also observe that for low pressure, Eq. (8) slightly overpredicts the value of the mean force, while for higher pressure the prediction well captures the data. While the mean value is insensitive to cohesion, the mean positive and negative normal forces, $\left\langle f_{\text {pos }}\right\rangle$ and $\left\langle f_{\text {neg }}\right\rangle$, as can be seen in Fig. 11, where we plot them against pressure for different values of Bo. Furthermore, the intensities of both negative and positive forces increase with increasing cohesion, which is in accordance with Fig. 8. Note that the mean positive (negative) force is linear with pressure and independent of cohesion below $\mathrm{Bo}=1$, while its dependence on pressure becomes nonlinear above $\mathrm{Bo}=1$. Though the origin of this nonlinearity is not clear, it is readily understood that cohesion enhances the collective motion of the particles, i.e., the particles rearrange less and the system is in a mechanically constrained state. Such constrain leads to increase in the magnitude of negative forces. As a consequence, positive forces also increase, in order to balance the negative ones. It is noteworthy that in Fig. 9, the increase of Bo increases the density of points in both positive and negative extremes, inside the shear band, in accordance with the previous considerations.

Similarly to what was observed for the mean force, cohesion seems not to affect the average number of contacts, as reported in Ref. [58], where we observed that cohesion had practically no effect on the contact number density (volumetric fabric). Figure 12 shows the fractions of repulsive and attractive contacts against pressure for different Bond numbers, normalized by the total number of contacts. An 

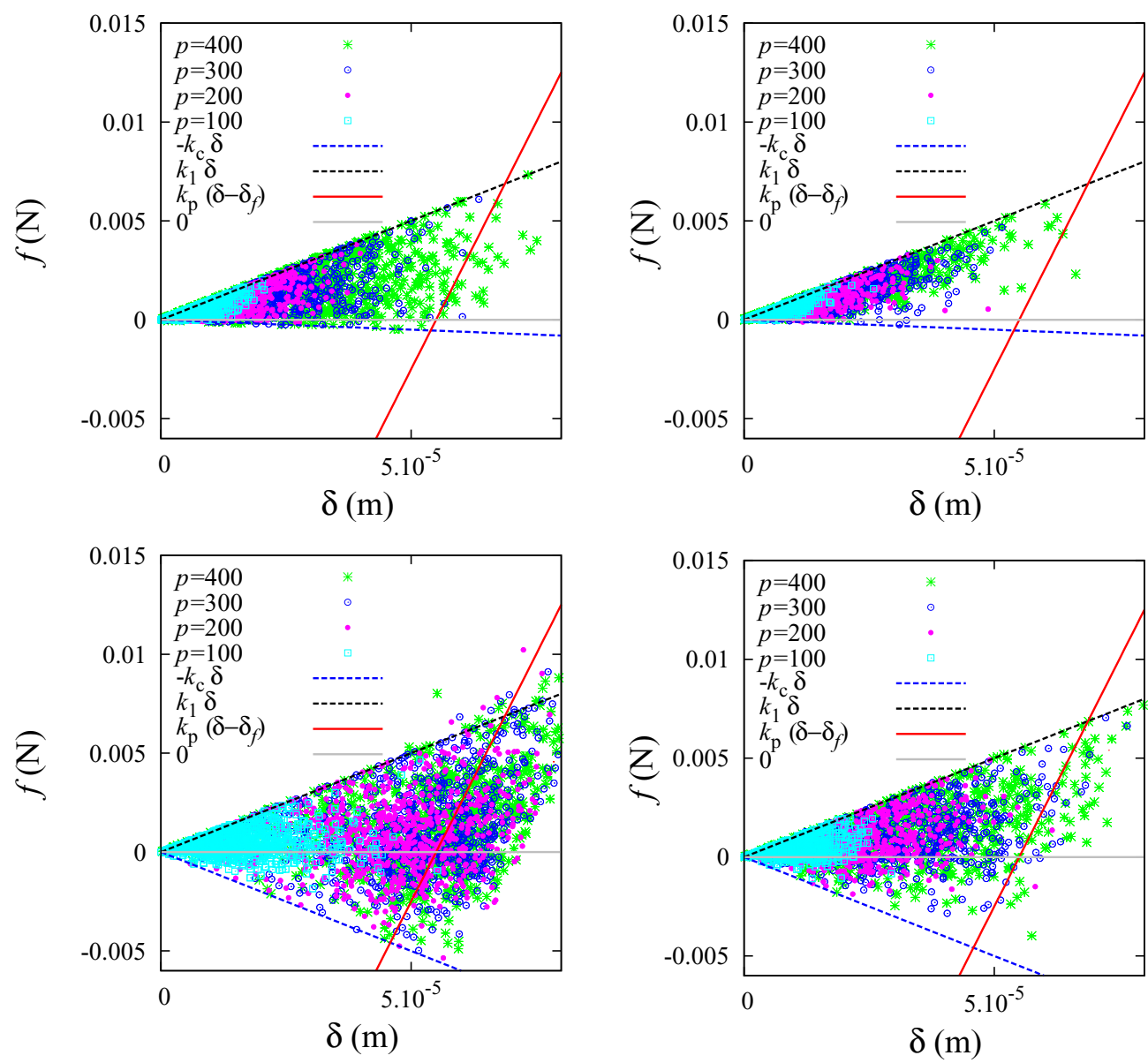

FIG. 9. (Color online) Scatter plots of overlaps and forces between all contacts inside (left) and outside (right) of the shear bands for different $\mathrm{Bo}=0.33$ and 2.85. The different symbols represent a zoom into the vertical ranges $z=8 \mathrm{~mm} \pm 1 \mathrm{~mm}$ (green stars), $15 \mathrm{~mm} \pm 1 \mathrm{~mm}$ (blue circles), $22 \mathrm{~mm} \pm 1 \mathrm{~mm}$ (magenta dots), and $29 \mathrm{~mm} \pm 1 \mathrm{~mm}$ (cyan squares), with approximate pressure as given in the inset. Note that the points do not collapse on the line $k_{p}\left(\delta-\delta_{f}\right)$ due to the finite width of the size distribution: Pairs of larger than average particles fall outside the indicated triangle. Radial range $0.075 \mathrm{~m} \leqslant r \leqslant 0.085 \mathrm{~m}$ (left) signifies data points inside the shear band, while the radial range $0.055 \mathrm{~m} \leqslant r \leqslant 0.065 \mathrm{~m}$ (right) signifies the data points outside the shear band.

increase in cohesion generates more attractive contacts while the number of repulsive contacts decrease. Interestingly, the

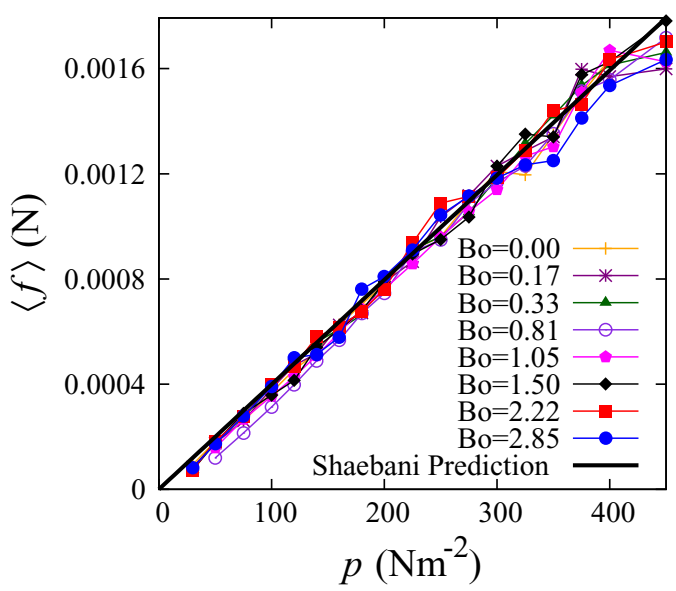

FIG. 10. (Color online) The mean normal force $\langle f\rangle$ inside the shear band plotted against pressure $p$, where different symbols represent the global Bond number (as given in the inset) and the solid line is given by Eq. (8). overall average coordination number remains independent of cohesion and contacts simply redistribute between the repulsive and attractive directions.

In contrast to the mean force, the mean overlap between particles in contact nonlinearly depends on cohesion, as shown in Fig. 13. In our model of cohesive particles [59], overlaps are always positive for both positive and negative forces. It is worth mentioning that for low Bo, $\langle\delta(t)\rangle$ saturates quickly, while for $\mathrm{Bo}=1.5,2.22,2.85$ it takes longer to reach the steady state due to the average plastic increase of the overlap [53].

\section{PDFs of forces and structures of strong force chains in shear bands}

The PDF of forces is also strongly affected by cohesion. Figure 14 shows the PDFs of normal forces in shear bands for different pressures and cohesion, where the forces are scaled by the mean normal force, i.e., $f^{*} \equiv f /\langle f\rangle$. As can be seen, the $\mathrm{PDF}$ of noncohesive particles $(\mathrm{Bo}=0)$ is almost independent of pressure [Fig. 14(a)], while it depends on pressure if the cohesive forces are very strong [Fig. 14(b)]. Figure 15 displays the variations of the PDFs for different intensities of 


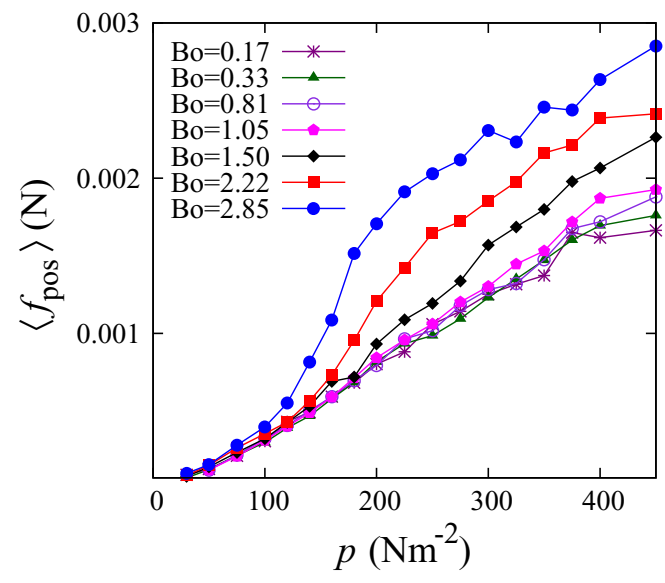

(a)

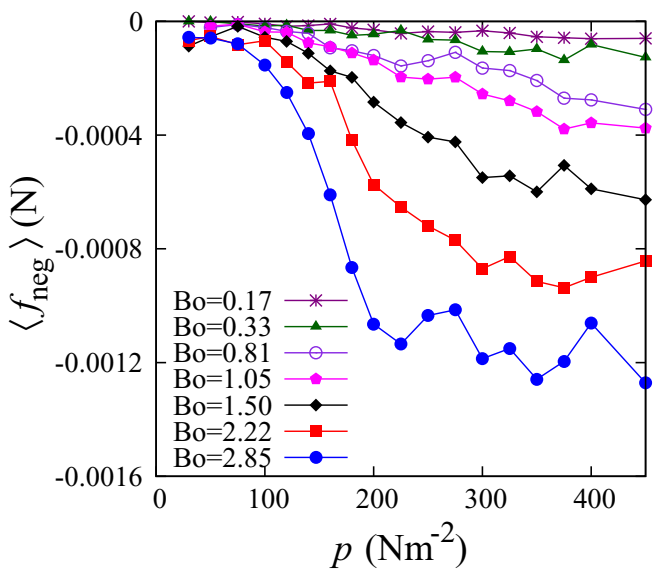

(b)

FIG. 11. (Color online) The mean (a) positive force $\left\langle f_{\text {pos }}\right\rangle$ and (b) negative force $\left\langle f_{\text {neg }}\right\rangle$ inside the shear band plotted against pressure $p$, where different symbols represent the global Bond number (as given in the inset).

cohesion, where we find that the PDF becomes broad with increasing cohesion and Bo $>1$. Therefore, strong cohesion, which leads the system to a mechanically constrained state induces larger fluctuations of positive and negative forces. We note that Yang et al. [51] also found similar trends in a static three-dimensional packing of small sized particles. They reported that the PDF gets broader with a decrease in particle size, i.e., with increasing cohesion. Broadening of the PDFs was also observed by Luding et al. [76] during cooling down of a sintered system.

The cohesive forces modify not only the shapes of the PDFs, but also their asymptotic behavior, i.e., the structure of strong force chains. The tails can be fitted by a stretched exponential function [77],

$$
P\left(f^{*}\right) \sim e^{-\left(f^{*} / f_{0}\right)^{\alpha}},
$$

with a characteristic force $f_{0}$ and a fitting exponent $\alpha$. Figure 16 displays the characteristic force and the exponent against the global Bond number Bo. If $\mathrm{Bo}<1$, we obtain $f_{0}=1.4 \pm 0.1$ and $\alpha=1.6 \pm 0.1$, which is very close to that predicted by Eerd et al. [77] for three-dimensional noncohesive

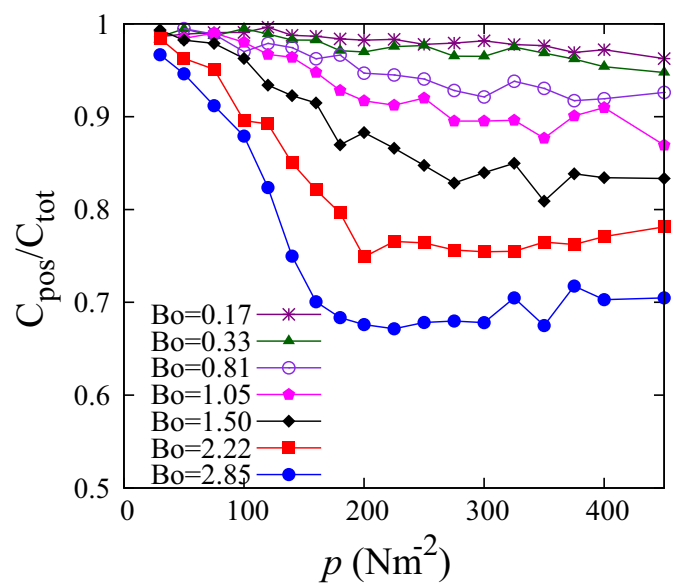

(a)

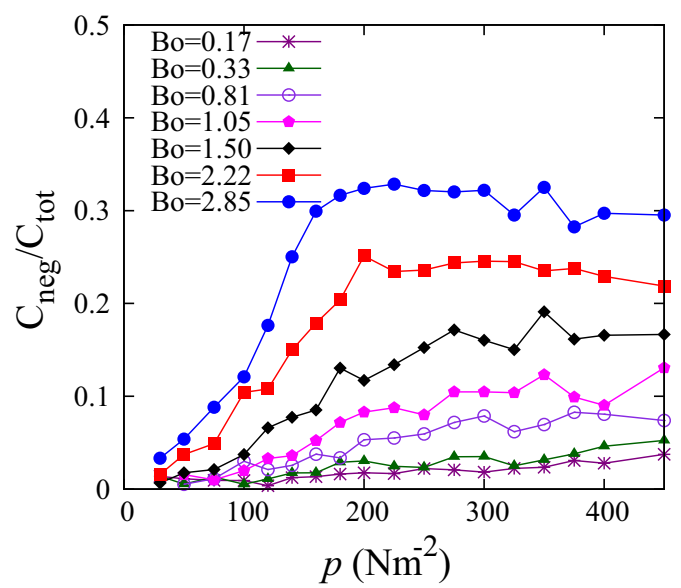

(b)

FIG. 12. (Color online) The fractions of (a) positive and (b) negative contacts inside the shear band plotted against pressure $p$, where different symbols represent the global Bond number (as given in the inset).

ensemble generated by MD simulations. However, for Bo $>1$, both characteristic force and fitting exponent decrease with increasing cohesion. The decreasing fitting exponent hints at

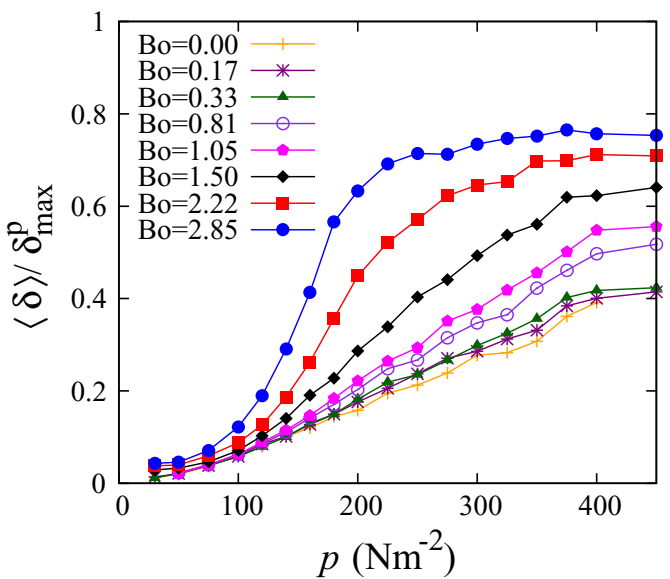

FIG. 13. (Color online) Normalized mean overlap $\frac{\langle\delta\rangle}{\delta_{\max }^{p}}$ inside the shear band plotted against pressure $p$, where different symbols represent the global Bond number (as given in the inset). 


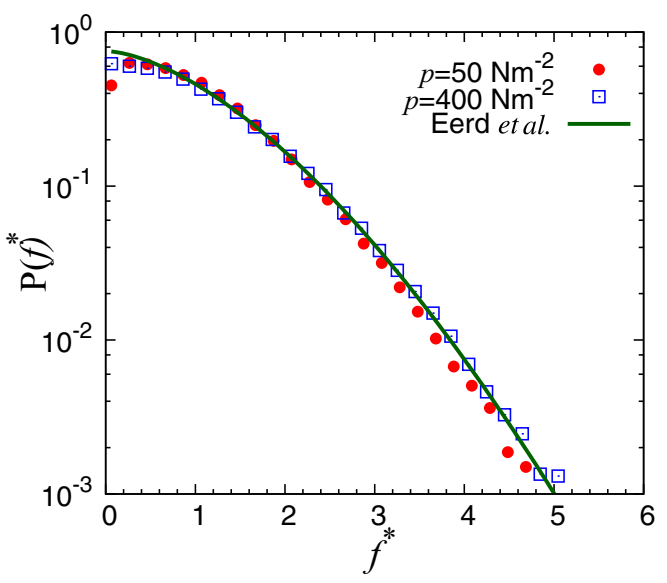

(a)

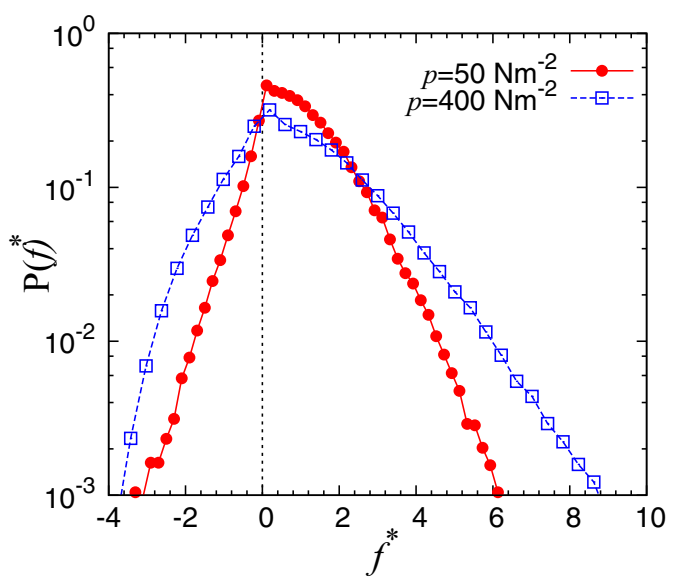

(b)

FIG. 14. (Color online) Probability distribution of the normalized force $f^{*}$ for (a) noncohesive $\mathrm{Bo}=0$ and (b) highly cohesive Bo $=2.85$ systems at different pressures $p$ in the system. Different symbols represent value of local pressure (as given in the inset).

stronger fluctuations in the force distribution. A Gaussian tail of the probability distribution would indicate a more homogeneous random spatial distribution of forces. The deviation towards an exponential distribution can be linked to an increase in heterogeneity in the spatial force distribution as mentioned in previous studies [78-80]. Therefore, we conclude that the tail of the PDF becomes a wider exponential with increasing cohesion, which implies a more heterogeneous spatial distribution, especially of the strong forces.

Finally, we observe that the fitting exponent decreases with increasing pressure, which implies that at high pressure where cohesion is more active due to the contact model the spatial distribution is more heterogeneous compared to low pressure.

\section{Anisotropy of force chain networks in shear bands}

In the case of simple shear as developed in the split-bottom shear cell, there are two nonzero eigenvalues of the strain rate tensor, which are equal in magnitude but opposite in sign, while the third eigenvalue is zero. The plane containing the eigenvectors associated to nonzero eigenvalues is called the shear plane, and the eigenvector with zero eigenvalue is

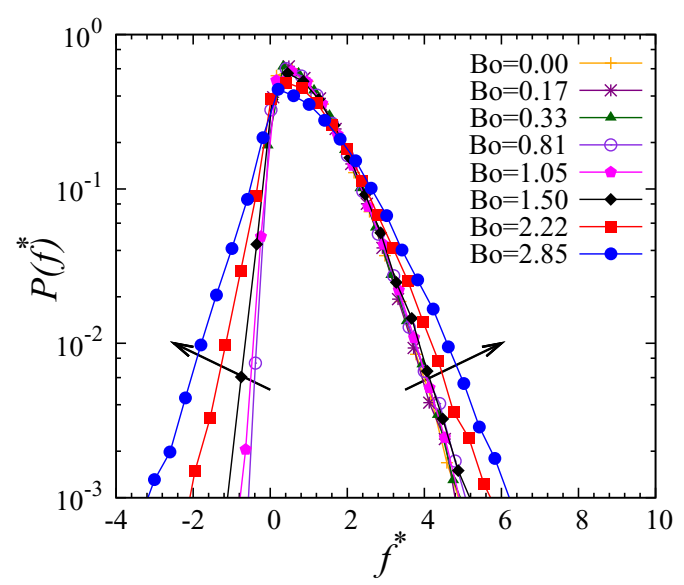

(a)

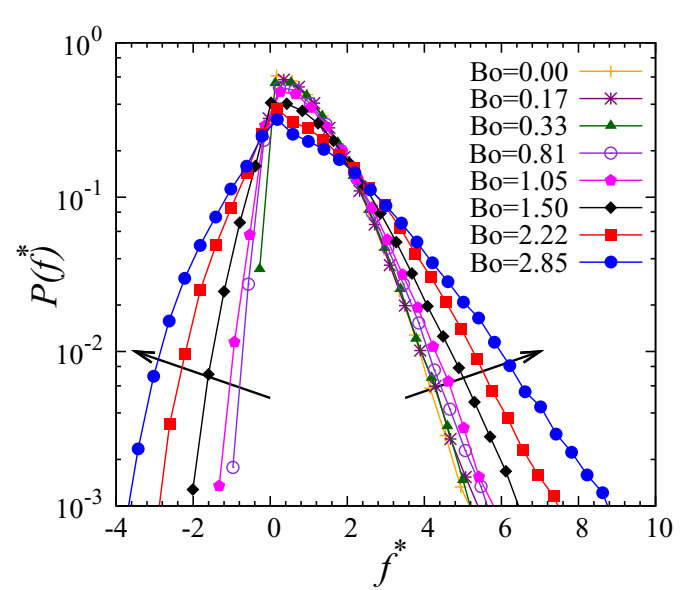

(b)

FIG. 15. (Color online) Probability distribution of normalized force $f^{*}$ for (a) low pressure $p=50 \mathrm{Nm}^{-2}$ (close to top) and (b) high pressure $p=400 \mathrm{Nm}^{-2}$ (close to bottom) in the system for data inside the shear band. Different symbols represent the global Bond number Bo (as given in the inset).

perpendicular to this plane (tangent to the shear band). In the following we will refer to the eigendirections associated to positive, negative, and zero eigenvalues as compressive, tensile, and neutral directions, respectively.

Note that the shear band here is not vertical, and, instead, its orientation changes with depth as shown by the schematic in Fig. 17. In this figure, the eigendirection of the neutral (zero) eigenvalue (green arrow) changes with height, along with the shear band. The neutral eigendirection defines the orientation of the shear plane (which is shown by the yellow regions). To extract the contacts aligned along these directions at a given pressure in the system, we first calculate the local tensor at a given strain rate and extract the three eigendirections $\mathbf{n}_{\gamma}^{\mathrm{i}}$ (with $i$ being compressive, neutral, and tensile). Next we search for contacts with the unit contact vector $\mathbf{n}_{\mathrm{c}}$ which, satisfy the condition $\left|\mathbf{n}_{c} \cdot \mathbf{n}_{\gamma}^{i}\right| \geqslant 0.9$. The contacts which satisfy the condition for the compressive eigendirection are termed compressive; tensile and neutral contacts are defined in a similar fashion. The forces carried by compressive, tensile, and neutral contacts are denoted by $f_{\text {com }}, f_{\text {ten }}$, and $f_{\text {neu }}$, respectively. 


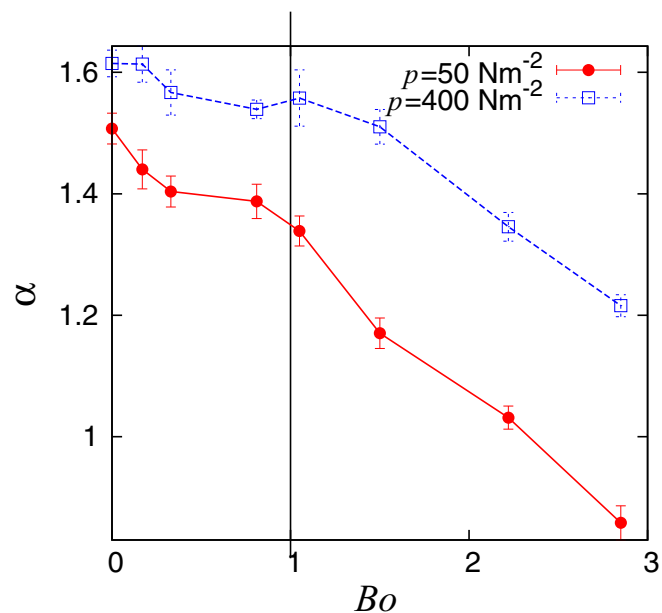

(a)

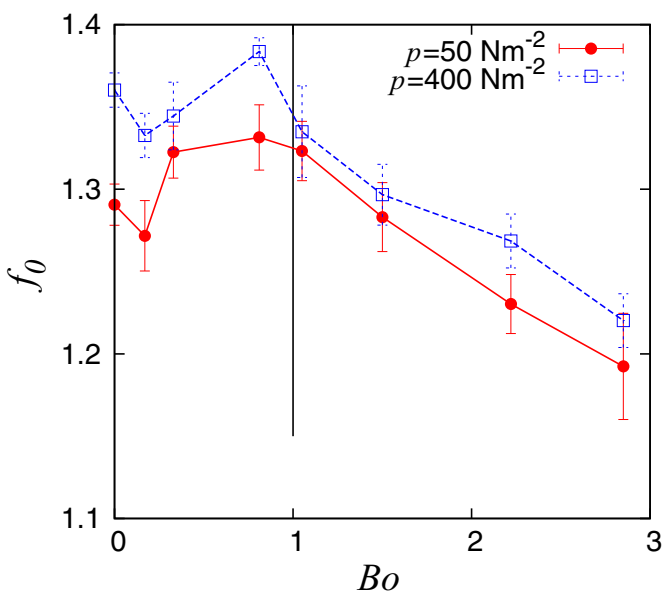

(b)

FIG. 16. (Color online) Fit parameters (a) $\alpha$ and (b) $f_{0}$ plotted against Bond number Bo. Different symbols represent value of local pressure (as given in the inset).

Since compressive and tensile directions are associated with loading and unloading of contacts, respectively, it is intuitive that in the absence of any external force other than shear, the mean force would be positive in the compressive direction, negative in the tensile direction, and almost zero in the neutral direction.

In our system, an external load-gravity-coexists with (external) shear. The neutral direction gets a contribution from the additional load only, while the two principal (compressive and tensile) directions get contributions from both shear and gravity. Because the cohesive force is activated by unloading, we expect that it affects the forces along the tensile direction.

Figure 18 shows the mean compressive, tensile, and neutral forces relative to the local mean force, $f_{\text {com } / \text { ten } / \text { neu }}^{\prime} \equiv$ $\left\langle f_{\text {com } / \text { ten } / \text { neu }}\right\rangle-\langle f\rangle$, plotted against pressure for different values of Bo. We find that $f_{\text {com }}^{\prime}(>0)$ and $f_{\text {ten }}^{\prime}(<0)$ are symmetric about zero and $f_{\text {neu }}^{\prime} \simeq 0$. The mean force along the neutral direction is independent of Bo, as the cohesion does not affect $f_{\text {neu }}$ due to the absence of shear (tension) in this direction. However, the direction-dependence increases with pressure

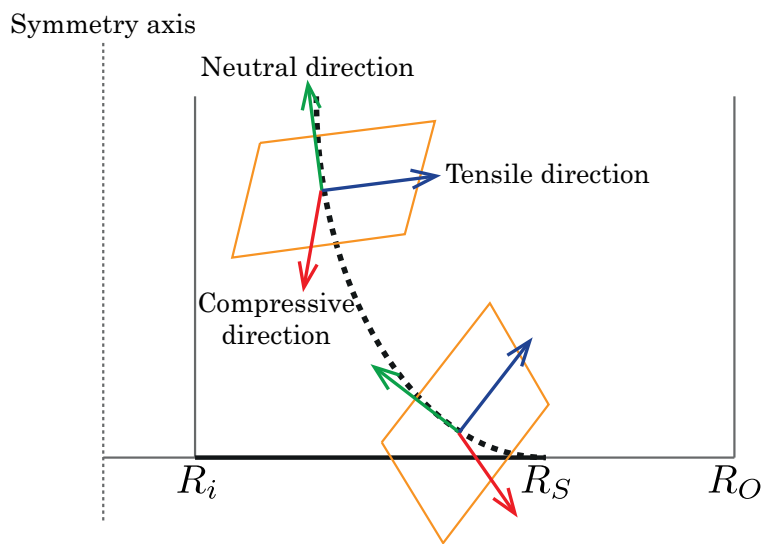

FIG. 17. (Color online) A sketch showing the shear band, shear plane, and three eigendirections of the strain rate tensor. Gray lines show inner and outer cylinders, while the solid brown line shows the split; the dashed black line shows the shear band which initiates at the split at the bottom and moves towards the inner cylinder as it moves towards the top. The green arrow represents the eigendirection for the neutral eigenvalue of the strain rate tensor, which is tangential to the shear band; perpendicular to this vector is the shear plane (yellow region), which contains the eigendirections for the compression (red arrow) and tensile (blue arrow) eigenvalues. $R_{i}, R_{s}$, and $R_{o}$ show the inner, split, and outer radii, respectively.

and cohesion. When $f_{\text {ten }}^{\prime}$ decreases $f_{\text {com }}^{\prime}$ increases in order to keep the mean overall force independent of cohesion. We point out here that the difference between forces carried by compressive and tensile contacts, i.e., the anisotropy of forces becomes more pronounced with increasing pressure and cohesion. This is consistent with the visual observation of force chains of negative and positive forces for different intensity of cohesion, as shown in Fig. 8.

Next, we study the PDFs of forces in the compressive, tensile, and neutral directions. Figure 19 displays the PDFs along each direction for noncohesive $\mathrm{Bo}=0$ and highly cohesive $\mathrm{Bo}=2.85$ systems, where the forces along different directions are normalized by the overall mean force. In a

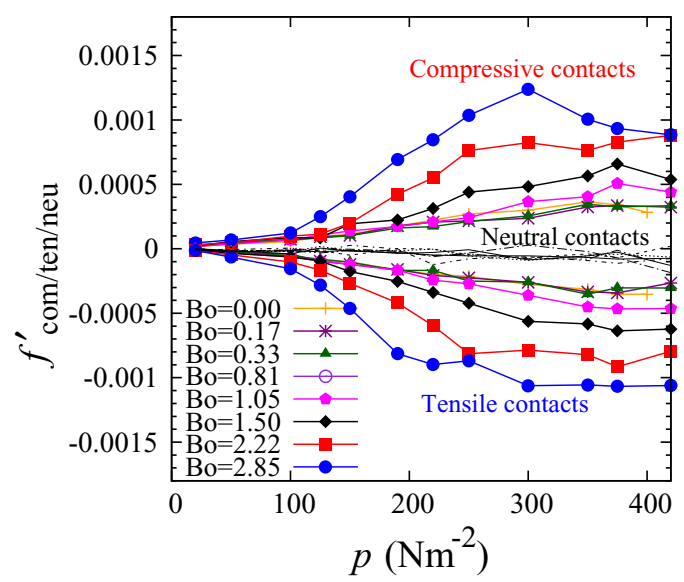

FIG. 18. (Color online) Mean forces in different eigendirections of the strain rate tensor, relative to the overall mean force plotted against the local pressure $p$ in the system. Different symbols represent the global Bond number Bo (as given in the inset). 


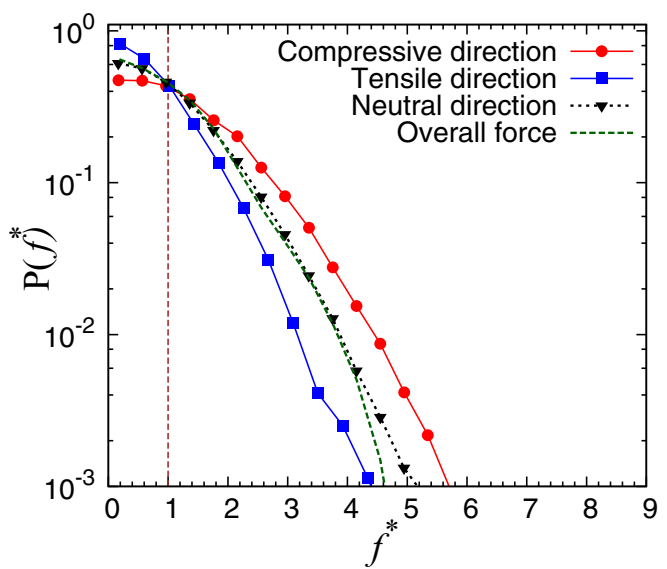

(a)

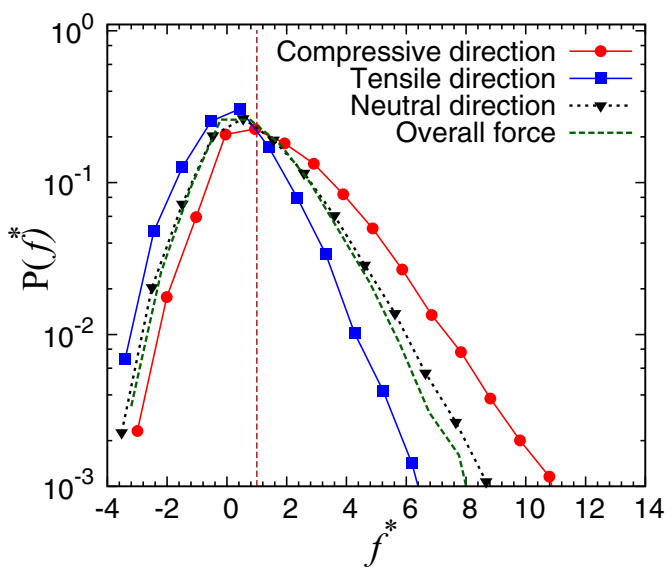

(b)

FIG. 19. (Color online) Probability distributions of normalized forces $f^{*}=f /\langle f\rangle$ in the compressive, tensile, and neutral directions inside the shear bands for high pressure in (a) noncohesive $\mathrm{Bo}=0$ and (b) highly cohesive Bo $=2.85$ systems. The dashed-line curves show the PDFs of the overall normalized forces, while the average force is indicated by the vertical lines.

noncohesive system [Fig. 19(a)], we observe that for weak forces, i.e., $f^{*}<1$, the PDF along the tensile direction is higher compared to that for the compressive direction. This is intuitive, as the majority of weak contacts will be aligned along the tensile direction. However, for $f^{*}>1$ the PDF along the compressive direction becomes higher compared to that along the tensile direction, as the majority of contacts along the compressive direction should carry strong forces [81]. For a highly cohesive system [Fig. 19(b)], a similar behavior is observed for strong positive forces $f^{*}>1$. While for weak positive and whole range of the negative forces, the PDF along the tensile direction is higher in comparison to the compressive direction. The PDFs of forces in the neutral direction lie between those in compressive and tensile directions, suggesting a close-to-average distribution of forces. It is interesting to note that both positive and negative forces are present in all directions. However, the positive and negative forces dominate in the compressive and tensile directions, respectively.

Figure 20 shows the variations of the PDFs along compressive and tensile directions for different values of Bo. If

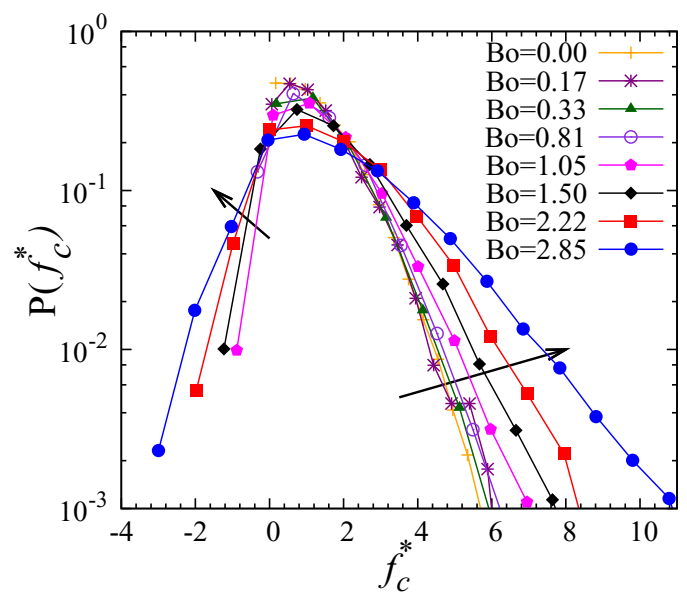

(a)

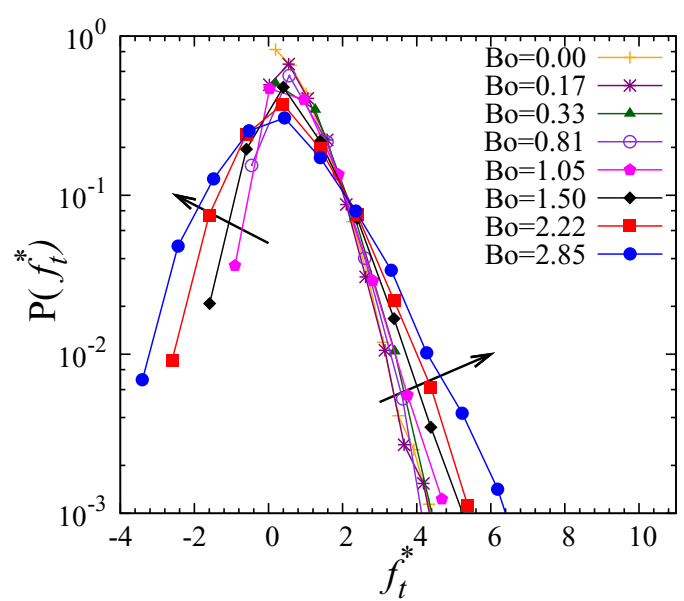

(b)

FIG. 20. (Color online) Probability distributions of normalized forces in (a) compressive $\left(f_{c}^{*}=f_{c} /\langle f\rangle\right)$ and (b) tensile $\left(f_{t}^{*}=\right.$ $\left.f_{t} /\langle f\rangle\right)$ directions inside the shear bands for high pressure. Different symbols represent different values of the global Bond number Bo as given in the inset.

Bo $<1$, the PDFs collapse on top of each other. However, the PDFs get wider with increasing cohesion above Bo $=1$. Such widening is more prominent for positive and negative forces in the compressive and tensile directions, respectively. Again, we confirm that strong cohesion leads to an increase of positive and negative forces in the compressive and tensile directions, respectively. Therefore, as the force distributions along the principal directions get more heterogeneous with increasing cohesion for Bo $>1$, the heterogeneity of the overall force structure increases.

Results in this section suggest that for low Bo, external load and shear dominate and govern the distribution of forces along compressive and tensile directions. The forces can adapt to external shear, and the particles rearrange and can avoid very large forces. In contrast, for high Bo, cohesion dominates over external forcing: The contact forces still respond to compression and tension, but their rearrangements are hampered by cohesion. Due to the sticky nature of cohesive forces, rearrangements of the contact network become more difficult, so 
very large contact forces as well as strong sticking forces occur together, leading to a more heterogeneous contact network.

\section{DISCUSSION AND CONCLUSION}

In this paper, we have studied the effect of cohesion on shear banding in dry cohesive powders. The global Bond number, Bo, can be used to quantify how strong cohesive forces are relative to the forces due to external compression, where Bo $\simeq 1$ very well predicts the transition from a free-flowing, noncohesive system to a cohesive system. Interestingly, many other features of the system also show a transition at Bo $\approx 1$. Using local Bo has no big advantage in this system but is recommended in general.

\section{A. Shear band}

Width and center position of the shear band are fairly unaffected by cohesion for Bo $<1$; only for Bo $\geqslant 1$ cohesion affects the flow behavior. Since cohesive forces tend to keep the particles in contact to stay connected for longer time; as consequence, for Bo $>1$, the velocity gradient decreases so that the width of the shear band increases with increasing cohesion. Cohesive forces assist the collective motion of particles, implying that attractive forces work against the localization of shear.

\section{B. Forces and their direction dependence}

The mean force $\langle f\rangle(p)$ (with $p \propto H-z$ ) is found to be independent of cohesion, just like the number of contacts. With increasing Bo, stronger attractive negative forces are possible at contacts (which is intuitive). However, these negative forces must be balanced by some stronger positive forces to maintain the same overall mean force.

Due to the planar shear that is established in the steady state, compressive and tensile contact forces are induced in compressive and tensile eigendirections of the local strain rate tensor, respectively, while along the third, neutral, direction neither compression nor tension take place. The mean force along the neutral direction remains unaffected by cohesion, which implies that cohesive forces in the system are activated by shear; more specifically, cohesive forces are activated by the tension in the respective (eigen-)direction. In other words, only about one-third of all contacts features considerable straininduced cohesion.

The mean force carried by contacts along compressive and tensile directions is symmetric about the mean overall force. For Bo $\leqslant 1$, this anisotropy of the force network is independent of cohesion, while for Bo $>1$ the anisotropy in the force network increases with cohesion. Macroscopically, this anisotropy in force is directly related to the shear stress; the trend in force anisotropy is very similar to the trends found in the shear stress in previous work [53].

\section{Force probability distribution}

Since granular systems are known to be heterogeneous in nature, we also analyzed the effect of cohesion on the force probability distributions. For noncohesive and weakly cohesive systems, no prominent effect of pressure on force distributions could be seen. For strong cohesion $\mathrm{Bo}>1$, pressure affects the distribution of forces by making the tails wider, and more symmetric, as compared to the cases with Bo $<1$. Splitting up the force distributions along the compressive and tensile directions reveals that, for higher Bo, cohesion broadens the force distributions along the tensile direction, which in turn affects the distribution along the compressive direction, which also becomes wider. This suggests an increase in heterogeneity in forces for Bo $>1$ along all directions, compressive, tensile, and neutral. For low Bo, the kinematics of shear helps the particles to rearrange and avoid very strong forces. In contrast, for high Bo, cohesion induces stickiness at the contacts so that rearrangements are suppressed, increasing the heterogeneity of the system, as evidenced by the wider tails of the probability distributions.

\section{Main message}

In conclusion, both the flow profiles (shear banding) and the force structure are unaffected by cohesion for Bo $<1$. In contrast, for Bo $\geqslant 1$, cohesion strongly affects the flow behavior, the anisotropy, and the internal force structure. Attractive forces thus reduce shear localization for $\mathrm{Bo}>1$ and promote heterogeneity of the force network. These two observations are consistent with previous studies with attractive forces concerning the rheology [30] and force structures for static packings [51].

As speculation, for a wider view, our results can be interpreted as follows: In the language of statistical mechanics, the global Bo corresponds to a control parameter and $\mathrm{Bo}=1$ to a critical point. The changes in the characteristic force and the fitting exponents show a weak pressure dependence, which might be better captured using a pressure-dependent, local Bond number. In our case, the macroscopic properties (position and width of the shear bands), the anisotropy, and the microstructural signatures (the tails of the PDFs) gradually increase for $\mathrm{Bo} \geqslant 1$. This continuous increase implies a second-order transition; however, confirming this would need a further detailed study. In addition, experiments performed with controlled, pressure-dependent cohesive strength would be exciting to confirm and validate our results. Finally, it would be interesting to reproduce our findings with different contact models, e.g., capillary bridges or simpler cohesive contact models with no pressure dependence.

\section{ACKNOWLEDGMENTS}

We thank M. Wojtkowski, N. Kumar, O. I. Imole, T. Weinhart, and Ruud van Ommen for stimulating discussions. Financial support (Project No. 07CJR06) through the "Jamming and Rheology" program of the Stichting voor Fundamenteel Onderzoek der Materie (FOM), which is financially supported by the "Nederlandse Organisatie voor Wetenschappelijk Onderzoek" (NWO), is acknowledged. K. Saitoh was funded by the NWO-STW VICI Grant No. 10828 .

\section{APPENDIX A: MAXIMUM ATTRACTIVE FORCE}

The extreme loading and unloading branches are reflected by the outer triangle in Fig. 1. Starting from a realized maximum overlap during loading, $\delta_{\max }<\delta_{\max }^{p}$, the unloading 
happens within the triangle, as can be characterized by a branch with stiffness,

$$
k_{2}=k_{1}+\left(k_{p}-k_{1}\right) \delta_{\max } / \delta_{\max }^{p}
$$

(as given in Ref. [58]). The elastic, reversible force along this branch is given by $k_{2}\left(\delta-\delta_{0}\right)[59,64]$. The intermediate stiffness $k_{2}$ follows from a linear interpolation between $k_{1}$ and $k_{p}$, as explained in Refs. $[59,64]$. The corresponding maximal attractive force is $f_{\mathrm{m}}=-k_{c} \delta_{\mathrm{m}}=-k_{c} \frac{\left(k_{2}-k_{1}\right)}{\left(k_{2}+k_{c}\right)} \delta_{\max }$. If we assume that the maximal overlap $\delta_{\max }^{p}$ is realized under a given external (compressive) pressure $p_{\max }$, then we can infer $\frac{p}{p_{\max }}=\frac{\delta_{\max }}{\delta_{\max }^{p}}$, with pressure $p$ being $p=k_{1} \delta_{\max } / A, A$ being a representative area. This leads to realized maximal attractive force being

$$
f_{\mathrm{m}}=-k_{c} \frac{\left(k_{2}-k_{1}\right)}{\left(k_{2}+k_{c}\right)} \frac{p}{p_{\max }} \delta_{\max }^{p} .
$$

Using Eq. (A1) in Eq. (A2), we get

$$
f_{\mathrm{m}}=-k_{c} \frac{\left(k_{p}-k_{1}\right) \frac{p_{\max }}{k_{1}}\left(\frac{p}{p_{\max }}\right)^{2}}{k_{c}+k_{1}+\left(k_{p}-k_{1}\right) \frac{p}{p_{\max }}} .
$$

This definition can be used to define a local Bond number as $\mathrm{Bo}_{l}^{a}(p)=f_{\mathrm{m}}(p) /\langle f(p)\rangle$, where mean force at that pressure is discussed in Sec. IIC. This Bond number is compared with various other definitions in Sec. II C.

\section{APPENDIX B: COHESIVE FORCE MAGNITUDE}

In order to get a feeling for the magnitude of the adhesion forces in experimental systems, we resort to Ref. [82] and estimate the attractive force as

$$
F_{\mathrm{vdW}}=\frac{H d}{24 l^{2}} \approx 1.7 \times 10^{-10} \mathrm{~N} \quad \text { or } \quad 1.7 \times 10^{-9} \mathrm{~N},
$$

for $\mathrm{SiO}_{2}$ particles with diameters $d=100 \mu \mathrm{m}$ or $10 \mu \mathrm{m}$ and Hamaker constant $H=6.6 \times 10^{-20} \mathrm{~J}$, minimal interparticle distance $l=s d \approx 4 \times 10^{-4} d$ (of the order of surface roughness for $9-\mu \mathrm{m}$ particles of high quality is actually a factor 10 smaller [83], while realistic roughness can be even larger; the value of $s$ is just a rough estimate). Due to this assumed relative magnitude of surface roughness, the adhesive force magnitude increases linearly with the (primary) particle diameter, while the gravitational force on the same particles,

$$
F_{g}=m g \approx 10^{-8} \mathrm{~N} \quad \text { or } 10^{-11} \mathrm{~N},
$$

decreases with the third power of the diameter, i.e., much faster. So while $0.1-\mathrm{mm}$-size particles are dominated by gravity, smaller $10-\mu$-size particles are dominated by their adhesion forces, as reflected by the respective (single-particle) gravity Bond numbers,

$\mathrm{Bo}_{g}=F_{\mathrm{vdW}} / F_{g}=\frac{H}{4 \pi \rho s^{2} d^{4}}=1.7 \times 10^{-2}$ or $1.7 \times 10^{2}$.
[1] J. Bridgwater, Géotechnique 30, 533 (1980).

[2] D. Howell, R. P. Behringer, and C. Veje, Phys. Rev. Lett. 82, 5241 (1999).

[3] P. Schall and M. van Hecke, Ann. Rev. Fluid Mech. 42, 67 (2010).

[4] G. Katgert, M. E. Möbius, and M. van Hecke, Phys. Rev. Lett. 101, 058301 (2008).

[5] S. Cohen-Addad, R. Höhler, and O. Pitois, Ann. Rev. Fluid Mech. 45, 241 (2013).

[6] G. Ovarlez, S. Rodts, X. Chateau, and P. Coussot, Rheol. Acta 48, 831 (2009).

[7] J. K. G. Dhont, M. P. Lettinga, Z. Dogic, T. A. J. Lenstra, H. Wang, S. Rathgeber, P. Carletto, L. Willner, H. Frielinghaus, and P. Lindner, Faraday Discuss. 123, 157 (2003).

[8] G. Mandl, L. Jong, and A. Maltha, Rock Mech. 9, 95 (1977).

[9] J. P. Bardet and J. Proubet, Géotechnique 41, 599 (1991).

[10] M. Oda and H. Kazama, Géotechnique 48, 465 (1998).

[11] D. R. Scott, Nature 381, 592 (1996).

[12] H.-B. Mühlhaus and I. Vardoulakis, Géotechnique 3, 271 (1987).

[13] M. Lätzel, S. Luding, and H. J. Herrmann, Granul. Matter 2, 123 (2000).

[14] M. Lätzel, S. Luding, H. J. Herrmann, D. W. Howell, and R. P. Behringer, Eur. Phys. J. E 11, 325 (2003).

[15] D. Fenistein and M. van Hecke, Nature 425, 256 (2003).

[16] S. McNamara and W. R. Young, Phys. Rev. E 53, 5089 (1996).

[17] S. McNamara and W. R. Young, Phys. Rev. E 50, R28 (1994).

[18] K. Kamrin and G. Koval, Phys. Rev. Lett. 108, 178301 (2012).

[19] D. L. Henann and K. Kamrin, Proc. Natl. Acad. Sci. USA 110, 6730 (2013).
[20] M. A. S. Quintanilla, A. Castellanos, and J. M. Valverde, Proc. Appl. Math. Mech. 3, 206 (2003).

[21] J. M. Valverde, M. A. S. Quintanilla, and A. Castellanos, Phys. Rev. Lett. 92, 258303 (2004).

[22] A. Castellanos, Adv. Phys. 54, 263 (2005).

[23] S. Herminghaus, Adv. Phys. 54, 221 (2005).

[24] C. Miclea, C. Tanasoiu, C. Miclea, F. Sima, M. Cioangher, and A. Gheorghiu, in Powders and Grains 2005 (Balkema, Leiden, Netherlands, 2005).

[25] L. Brendel, J. Török, R. Kirsch, and U. Bröckel, Granul. Matter 13, 777 (2011).

[26] A. Hatzes, F. Bridges, D. Lin, and S. Sachtjen, Icarus 89, 113 (1991).

[27] F. Spaepen, Acta Meta. 25, 407 (1977).

[28] J. Li, F. Spaepen, and T. C. Hufnagel, Philos. Mag. A 82, 2623 (2002).

[29] L. Bécu, S. Manneville, and A. Colin, Phys. Rev. Lett. 96, 138302 (2006)

[30] P. Chaudhuri, L. Berthier, and L. Bocquet, Phys. Rev. E 85, 021503 (2012).

[31] J. Vermant, Curr. Opin. Coll. Interface Sci. 6, 489 (2001).

[32] R. Höhler and S. Cohen-Addad, J. Phys.: Condens. Matter 17, R1041 (2005)

[33] P. Coussot and G. Ovarlez, Eur. Phys. J. E 33, 183 (2010).

[34] N. Estrada, A. Lizcano, and A. Taboada, Phys. Rev. E 82, 011303 (2010).

[35] R. Mani, D. Kadau, D. Or, and H. J. Herrmann, Phys. Rev. Lett. 109, 248001 (2012).

[36] R. Schwarze, A. Gladkyy, F. Uhlig, and S. Luding, Granul. Matter 15, 455 (2013). 
[37] J. Yuan, Q. Zhang, B. Li, and X. Zhao, Bull. Eng. Geol. Environ. 72, 107 (2013).

[38] C. H. Liu, S. R. Nagel, D. A. Schecter, S. N. Coppersmith, S. Majumdar, O. Narayan, and T. A. Witten, Science 269, 513 (1995).

[39] D. M. Mueth, H. M. Jaeger, and S. R. Nagel, Phys. Rev. E. 57, 3164 (1998).

[40] G. Løvoll, K. J. Måløy, and E. G. Flekkøy, Phys. Rev. E 60, 5872 (1999).

[41] D. L. Blair, N. W. Mueggenburg, A. H. Marshall, H. M. Jaeger, and S. R. Nagel, Phys. Rev. E 63, 041304 (2001).

[42] T. S. Majmudar and R. P. Behringer, Nature 435, 1079 (2005).

[43] K. Liffman, D. Y. C. Chan, and B. D. Hughes, Powder Technol. 72, 255 (1992).

[44] F. Radjai, D. E. Wolf, M. Jean, and J.-J. Moreau, Phys. Rev. Lett. 80, 61 (1998).

[45] L. E. Silbert, G. S. Grest, and J. W. Landry, Phys. Rev. E 66, 061303 (2002).

[46] J. H. Snoeijer, M. van Hecke, E. Somfai, and W. van Saarloos, Phys. Rev. E 67, 030302 (2003).

[47] L. E. Silbert, Soft Matter 6, 2918 (2010).

[48] V. Trappe, V. Prasad, L. Cipelletti, P. N. Segre, and D. A. Weitz, Nature 411, 772 (2001).

[49] V. Richefeu, Moulay Saï El Youssoufi, and F. Radjaï, Phys. Rev. E 73, 051304 (2006).

[50] F. A. Gilabert, J.-N. Roux, and A. Castellanos, Phys. Rev. E 75, 011303 (2007).

[51] R. Y. Yang, R. P. Zou, A. B. Yu, and S. K. Choi, Phys. Rev. E 78, 031302 (2008).

[52] F. Radjai, V. Topin, V. Richefeu, C. Voivret, J. Delenne, E. Azéma, and S. El Youssoufi, AIP Conf. Proc. 1227, 240 (2010).

[53] S. Luding and F. Alonso-Marroquín, Granul. Matter 13, 109 (2011).

[54] S. T. Nase, W. L. Vargas, A. A. Abatan, and J. McCarthy, Powder Technol. 116, 214 (2001).

[55] P. G. Rognon, J. Roux, and M. Naam, J. Fluid Mech. 596, 21 (2008).

[56] M. P. Allen and D. J. Tildesley, Computer Simulation of Liquids (Oxford University Press, Oxford, 1987).

[57] P. A. Cundall, in Proceedings of the International Symposium on Rock Fracture, October 1971 (International Society for Rock Mechanics (ISRM), Nancy, France, 1971), Vol. 1, paper no. II-8, pp. 129-136.

[58] A. Singh, V. Magnanimo, and S. Luding, AIP Conf. Proc. 1542, $682(2013)$
[59] S. Luding, Granul. Matter 10, 235 (2008).

[60] J. Tomas, Granul. Matter 6, 75 (2004).

[61] C. Thornton and Z. Ning, Powder Technol. 12, 154 (1998).

[62] S. C. Thakur, H. Ahmadian, J. Sun, and J. Y. Ooi, Particuology 12, 2 (2013).

[63] M. Pasha, S. Dogbe, C. Hare, A. Hassanpour, and M. Ghadiri, Granul. Matter 16, 151 (2014).

[64] A. Singh, V. Magnanimo, and S. Luding, Powder Technology (unpublished).

[65] D. Fenistein, J. W. van de Meent, and M. van Hecke, Phys. Rev. Lett. 92, 094301 (2004).

[66] S. Luding, Particul. Sci. Technol. 26, 33 (2007).

[67] S. Luding, Particuology 6, 501 (2008).

[68] J. A. Dijksman and M. van Hecke, Soft Matter 6, 2901 (2010).

[69] T. Unger, J. Török, J. Kertész, and D. E. Wolf, Phys. Rev. Lett. 92, 214301 (2004).

[70] D. Fenistein, J.-W. van de Meent, and M. van Hecke, Phys. Rev. Lett. 96, 118001 (2006)

[71] A. Ries, D. E. Wolf, and T. Unger, Phys. Rev. E 76, 051301 (2007).

[72] F. da Cruz, S. Emam, M. Prochnow, J.-N. Roux, and F. Chevoir, Phys. Rev. E 72, 021309 (2005).

[73] G. Ovarlez, S. Rodts, A. Ragouilliaux, P. Coussot, J. Goyon, and A. Colin, Phys. Rev. E 78, 036307 (2008).

[74] X. Wang, H. P. Zhu, S. Luding, and A. B. Yu, Phys. Rev. E 88, 032203 (2013).

[75] M. R. Shaebani, M. Madadi, S. Luding, and D. E. Wolf, Phys. Rev. E 85, 011301 (2012).

[76] S. Luding, K. Manetsberger, and J. Müllers, Jour. Mech. and Phys. Solids 53, 455 (2005).

[77] A. R. T. van Eerd, W. G. Ellenbroek, M. van Hecke, J. H. Snoeijer, and T. J. H. Vlugt, Phys. Rev. E 75, 060302 (2007).

[78] F. Radjai, M. Jean, J.-J. Moreau, and S. Roux, Phys. Rev. Lett. 77, 274 (1996)

[79] H. A. Makse, D. L. Johnson, and L. M. Schwartz, Phys. Rev. Lett. 84, 4160 (2000).

[80] H. P. Zhang and H. A. Makse, Phys. Rev. E 72, 011301 (2005).

[81] B. P. Tighe, J. E. S. Socolar, D. G. Schaeffer, W. G. Mitchener, and M. L. Huber, Phys. Rev. E 72, 031306 (2005).

[82] M. Tahmasebpoora, L. de Martin, M. Talebi, N. Mostoufia, and J. R. van Ommen, Phys. Chem. Chem. Phys. 15, 5788 (2013).

[83] R. Fuchs, T. Weinhart, J. Meyer, H. Zhuang, T. Staedler, X. Jiang, and S. Luding, Granul. Matter 16, 281 (2014). 